\title{
Analysis of face stability of circular tunnel in purely cohesive soils driven by
}

\section{shield}

\author{
${ }^{1}$ Jinhui Liu, ${ }^{2,3}$ Wantao Ding, ${ }^{4}$ Mingbin Wang \\ ${ }^{1}$ School of civil engineering, Shandong Jiaotong University, Jinan, China \\ 2. Research Center of Geotechnical and Structural Engineering, Shandong University, Jinan, China. \\ 3. School of Qilu Transportation, Shandong University, Jinan, China. \\ 4. School of Civil Engineering, Ludong University, Yantai, China.
}

Abstract: Based on the kinematic approach of the limit analysis and slip-line theories, this paper proposes a new $2 \mathrm{D}$ analytical model to evaluate the collapse support pressure to ensure the face stability of a circular tunnel in purely cohesive soils driven by a shield. The normality conditions, the yield criterion and the vertical soil arching effect are considered in the analytical model. Two upper bound solutions corresponding to the ratio of the cover to the diameter (C/D) are derived from considering the mechanisms based on the motion of rigid multi-blocks. Comparisons are made with existing upper and lower bound solutions published in previous articles. The results are close to the solutions of practical engineering. The failure mechanisms proposed in this study provide a better explanation for the failure process in the heading of the tunnel face.

Key words: heading; stability; limit analysis; plane strain; slip-line

\section{Introduction}

The advanced shield techniques (earth, slurry or air shield) are widely used to construct a shallow tunnel in soft ground. Nevertheless, if the support pressure acting on the tunnel face is not sufficient to balance the external earth and water pressure, face collapse might occur. Hence, the failure mechanisms and the limit support pressures continue to be an important research issue in stability analysis of the tunnel face.

The face stability of shallow circular tunnels driven in cohesive soils has been investigated by several authors. Based on laboratory extrusion tests and field observations, Broms and Bennermark (1967) first defined a stability ratio $N$, which is expressed as 
$N=\left[\sigma_{s}-\sigma_{T}+\gamma \cdot(C+D / 2)\right] / c_{u}$, where $\sigma_{s}$ is the possible surcharge loading on the ground surface, $\sigma_{T}$ is the uniform pressure applied to the tunnel face, $\gamma$ is the soil unit weight, $C$ is the depth of cover, $D$ is the diameter of the circular tunnel, and $c_{u}$ is the soil undrained cohesion. Subsequently, Davis et al.(1980) proposed a more theoretical approach derived from the limit state design concept. Schofield(1980) conducted a centrifuge model test and proposed a general expression for the stability ratio $N$ that is dependent on tunnel depth. Kimura and Mair (1981) performed a centrifuge test and noted that the limit support pressure depends on the tunnel cover for $5<N<10$. Idinger et al.(2011) investigated the face stability of shallow tunnel using the centrifuge model test and reported the arching effects of soil occurring at an overburden ratio of $\mathrm{C} / \mathrm{D}=1.0$. Based on a limit equilibrium analytical method, Ellstein(1986) presented an analytical solution for $N$ for homogeneous cohesive soils, which verified the results of Kimura and Mair (1981). Based on classical plasticity theory, Augarde et al.(2003) derived rigorous bounds on load parameters using the finite element limit analysis method and reported that the stability ratio $N$ used to analyse the stability of an idealized heading in undrained soil conditions is not rigorous. Lee et al.(2006) carried out a series of centrifuge model tests and numerical simulations of these tests to study the tunnel stability and arching effects that develop during tunnelling in soft clayey soil and proposed the boundaries of the positive and negative arching zones. More recently, the kinematic methods in the field of limit analysis using continuous velocity fields have been proposed by researchers. Based on an admissible continuous velocity field obtained directly from elasticity theory, Klar et al.(2007) investigated the face stability analysis of circular tunnels in purely cohesive soils and proposed 2D and 3D upper-bound solutions for this problem. Mollon et al.(2013) proposed two new continuous velocity fields to analyse the collapse of tunnel face and noted that these velocity fields agreed with the actual failures observed in undrained clay.

For the face stability analysis of shallow tunnels, the rigid multi-blocks failure mechanisms were often adopted to solve the collapse pressure of the tunnel face. Davis et al.(1980) derived upper and lower bound stability solutions for collapse under undrained conditions. Leca et al.(1990) obtained three upper bound solutions from the consideration mechanisms based on the motion of rigid conical blocks in a frictional material. Mollon et al.(2009,2010,2011) improved these mechanisms and 
obtained solutions relatively close to those of real projects in cohesive and frictional soils. More recently, several authors adopted numerical and experimental methods to evaluate the collapse pressure and proposed results closer to those of actual situations. Chen et al.(2011,2013) analysed the face stability of shallow shield tunnels in dry sands using 3D DEM and 3D FDM and proposed a two-stage failure pattern based on the observation of earth pressure. Based on the results of smallscale model tests under normal gravity $(1 \mathrm{~g}), \operatorname{Kirsch}(2010)$ proposed that the overburden and the initial soil density do not influence the required support pressure. Considering the seepage force around the tunnel face, Lu et al.(2014) investigated the relationship between the support pressure and displacement of the shield tunnel face using a 3D FEM. For layered soils, Senent et al.(2015) improved the model proposed by Senent et al.(2013) to analyse the possibility of a partial collapse occurring on the tunnel face. Zhang et al.(2013,2015) developed a 2D DEM model to analyse the behaviours of cohesive-frictional soils when tunnelling using a slurry shield and proposed a new 3D analytical model with four truncated cones on which a distributed force acts based on the results of numerical simulations.

This study aims at a face stability analysis of the purely cohesive soils in the framework of the kinematic approach of limit analysis theory. The rigid block failure mechanisms are a simple and intuitive approach and are either translational or rotational. Though the shapes of the blocks in the failure mechanisms well satisfy the normality condition proposed by Chen(1975), they may conflict with the yield criterion of the soils. Many three- dimensional analytical models and numerical simulations have also been constructed to analyse this problem using the finite element limit analysis method. However, the progressive failure of the tunnel face cannot be explained using these methods. While the problem of tunnel face stability is inherently three-dimensional, much can be learned from the behaviour of a reasonable two-dimensional model. Therefore, considering the changes in the soil pressure state and the influence of the vertical soil arching effect in the failure zone, we proposed a new 2D failure mechanism and constructed an admissible continuous velocity field to analyse the critical collapse face pressure of purely cohesive soils using the slip-line and limit analysis theories.

\section{Slip-line and limit analysis theories}

\section{Slip-line theory}

The yield condition of the soils is often evaluated using the Coulomb criterion. Considering both 3 
the equations of equilibrium and the yield condition, a set of solutions of the plastic equilibrium in the yielded zone can be developed. Combined with the stress boundary conditions, the stresses in the yielded zone in front of the tunnel face can be studied using these solutions. For specific problems, the slip-line field can be established using the slip-line method, which means that the stress-strain relationship of the soil is ignored, and only the equilibrium and yield conditions are considered. Thus, only a partial stress field region is constructed, and occasionally, the solution can be invalid outside the partial stress field region. To solve this problem, a reasonable extended stress field is constructed in this study. Therefore, considering the theory of limit analysis, accurate solutions for the collapse face pressures of the tunnel can be obtained.

\section{Limit analysis theory}

In the limit analysis, the soil is idealized as a perfectly elastic-plastic material that obeys normality conditions (or the associated flow rules). Based on the limit analysis, the associated theorems are established. Much experimental evidence validates that this assumption is reasonable for many clays. Thus, the critical collapse pressure of a tunnel face can be examined theoretically using the upperbound theorems for purely cohesive soils. The upper-bound theorem states that if a kinematically admissible velocity field can be found and if uncontained plastic flow previously occurred, then the deduced loads will be higher than or equal to those associated with collapse.

The aim of this study is to use the kinematic method of the limit analysis to study the face stability of purely cohesive soils. As the internal friction angle $\phi$ of purely cohesive soils is zero, plastic deformation in a purely cohesive soil develops without any volume change and satisfies the normality condition. Thus, the failure surfaces are assumed to follow the stress characteristics, which are also the velocity characteristics for purely cohesive soils. The actual sliding surface is characterized by a velocity and a stress.

\section{Admissible continuous translational velocity field}

The yield condition of the soils is often evaluated using the Coulomb criterion. A purely cohesive soil can be regarded as a rigid plastic Tresca material, as its internal friction angle $\varphi$ is zero. Thus, the Mohr circle of stress of a purely cohesive soil is shown in Figure 1. 


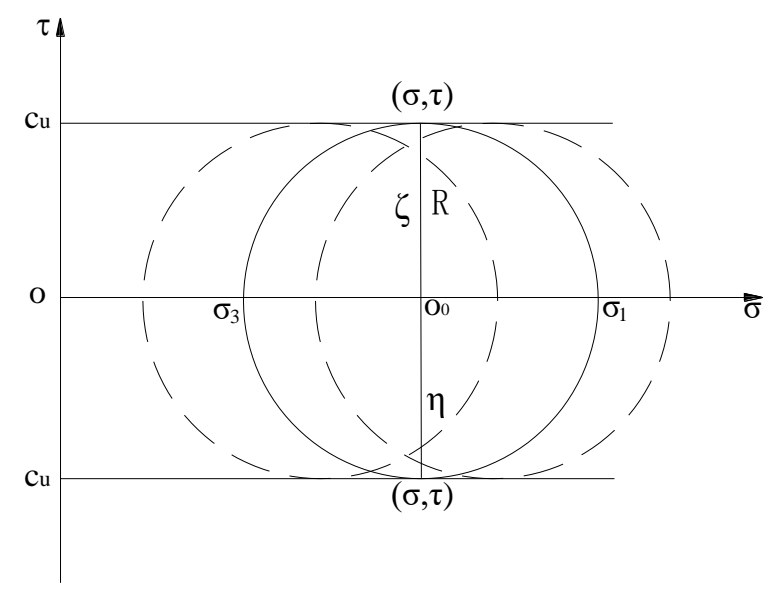

Fig. 1 Mohr circle of stress of purely cohesive soil

Based on the slip-line theory, when the material is in the plastic state, two orthogonal shear planes exist at each point for plane strain problems, as shown in Figure 2.

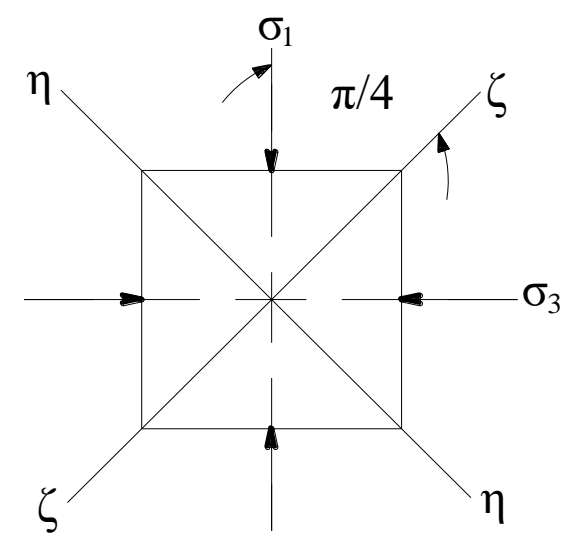

Fig. 2 Two possible shear planes at a point

This study focuses on the reasonable failure mechanism and the collapse critical pressure of the shield tunnel under the plane strain heading (as shown in Figure 3) for purely cohesive soils. Furthermore, the stress characteristics and the velocity characteristics are assumed to be identical.

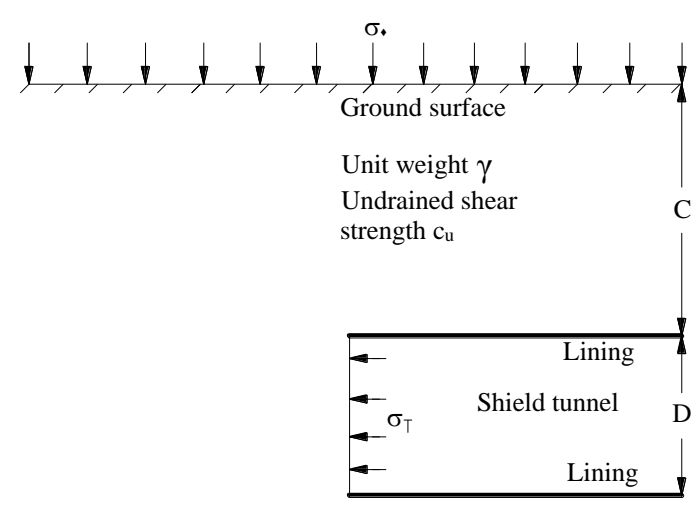

Fig. 3 The plane strain heading 
To construct a reasonable stress field, that is, an admissible continuous velocity field, the following assumptions are made.

(a) There is no shear force on the tunnel face;

(b) The effect of the Terzaghi level arching effect on the upper soils of the lining in the rear of the tunnel face is ignored when $C / D \leq 0.5$, where $C$ is the depth of cover, and $D$ is the tunnel diameter;

(c) The soils at the top of the tunnel face is in the plastic state when tunnelling, that is, $\sigma_{s} / c_{u}+\gamma C / c_{u} \geq 2$, where $\sigma_{s}$ is the possible surcharge loading acting on the ground surface, $\gamma$ is the soil unit weight, $C$ is the depth of cover, and $c_{u}$ is the soil undrained cohesion.

Based on these assumptions, a new 2D failure mechanism is proposed, as shown in Figure 4. The level of soil stress in the front of the tunnel face is analysed.

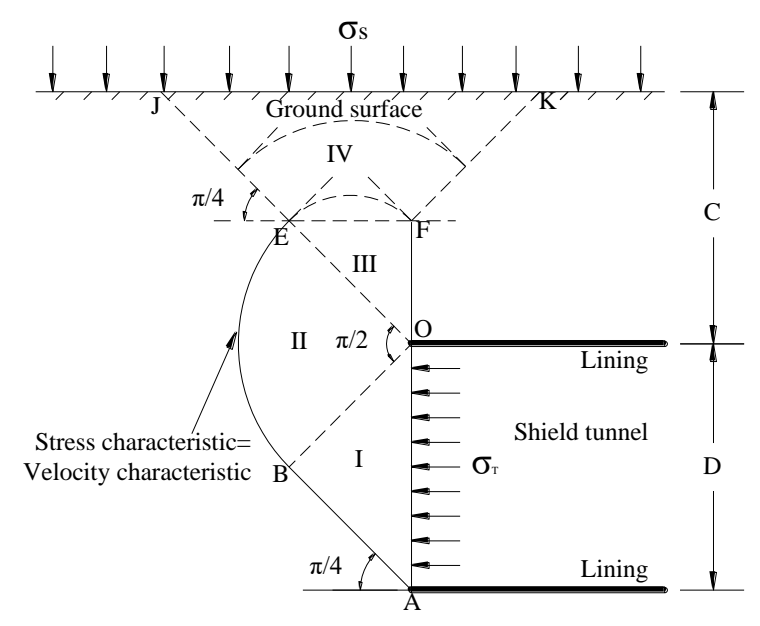

Fig. 4 A new 2D failure mechanism

The mechanism consists of four zones, i.e., Zone I, II, III and possibly IV. For failure zone I, when the support pressure $\sigma_{\mathrm{T}}$ acting on the tunnel face is not sufficient to ensure the stability of the tunnel face, the tunnel face can be regarded as the moveable vertical surface of failure zone I. The soil in zone $\mathrm{I}$ is in the limit state of the active earth pressure. Thus, zone I is a Rankine zone. For zone II, as the soils in zone II are constrained by the soils in zone I, zone III, and the elastic area in front of zone II, the stress principal axis in zone II should deflect to different degrees based on the associative flow rule(see.Chevalier,2013). Furthermore, the soils in zone II are in the plastic state 
and satisfy the Tresca yield criterion. Hence, zone II is a transition zone formed by a circular arc with radius $\sqrt{2} D / 2$ and two adjacent boundaries (see Chen, 1975). Due to the assumption that the soils behind line EF are stable (i.e., the Terzaghi horizontal arc effect is ignored), deflection of the stress principal axis does not occur in zone III, in which the soils are in the plastic state. Therefore, zone III is also a Rankine zone. Zone IV is a possible failure zone. When the soils in zone IV are in a plastic state, zone IV can be regarded as a Rankine zone but is affected by the vertical soil arching effect occurring at the top of zone III. It must be determined whether a failure area appears in zone IV.

Based on the slip-line and limit analysis theories, together with considering the normality conditions and Tresca yield criterion, we construct an admissible continuous velocity field, as shown in Figure 5. In Figure 5, the line $\mathrm{AB}$ and the arc $\mathrm{BE}$ represent the maximum shear stress trajectory of the soils and are the stress boundaries of the plastic and elastic soil regions. The lines EJ and FK are the potential trajectories of the maximum shear stress when the soil in the yielding zone IV. Moreover, the stability of soils in zone IV needs to be assessed.

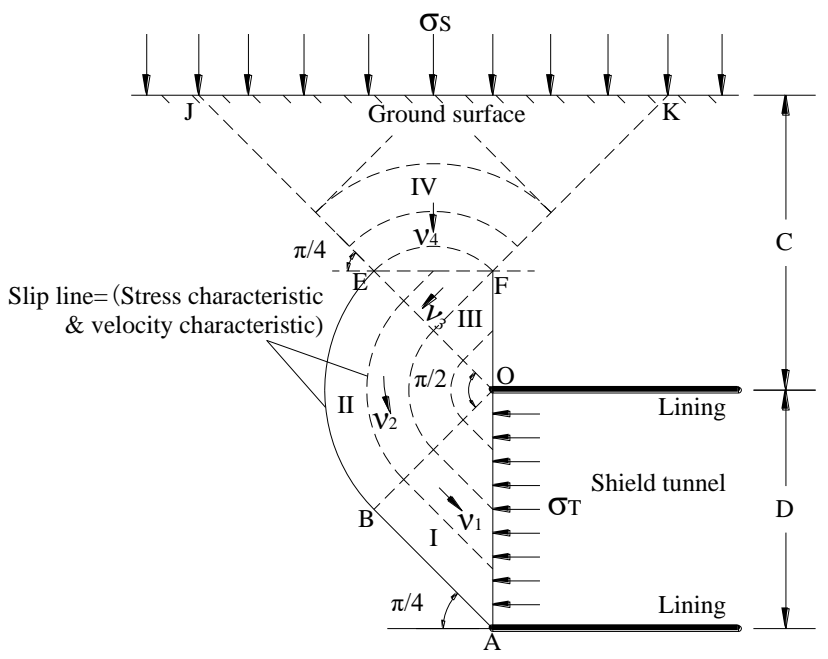

Fig. 5 Admissible velocity field

In this study, the motion of the soils in failure zones I, II and III is assumed to be translational. The fracture zone IV is assumed to respect Terzaghi's theory of relative soil pressure. Thus, a kinematically admissible velocity field is constructed, as shown in Figure 6; the admissible displaced pattern is shown in Figure 7. 


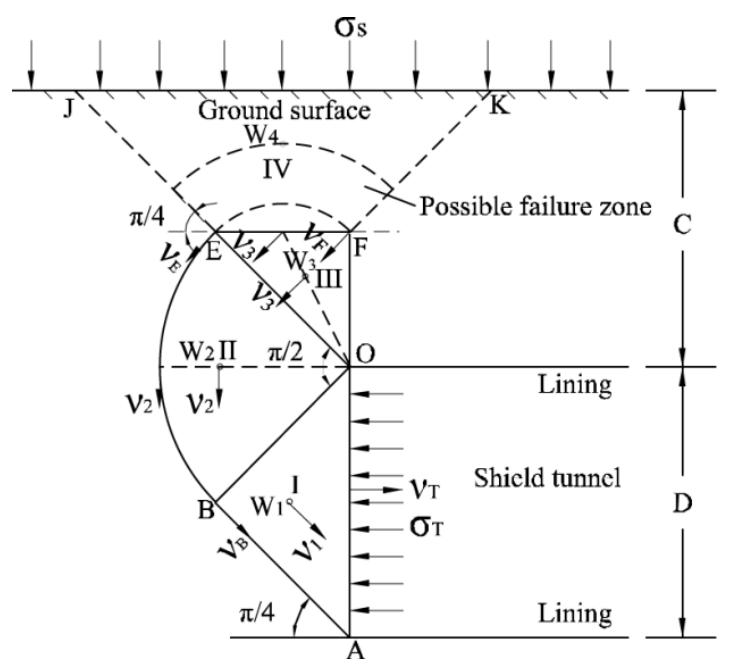

Fig. 6 Kinematically admissible velocity field

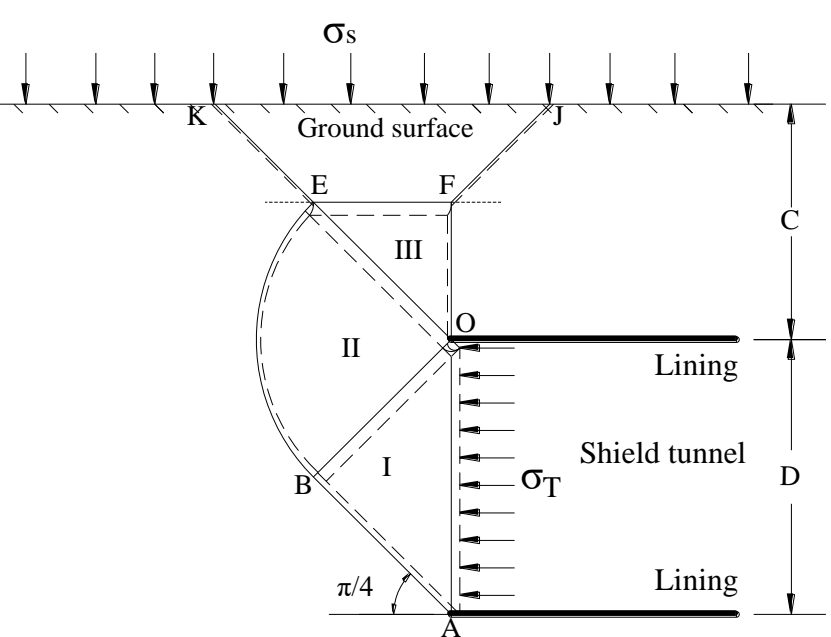

Fig. 7 Admissible displaced pattern

The kinematic velocity magnitude of the possible failure zones, i.e., zone I, II, and III, can be expressed as follows:

$v_{1}=v_{2}=v_{3}(1)$

The kinematic velocity magnitude at points $\mathrm{B}, \mathrm{E}$ and $\mathrm{F}$ is given by the following:

$v_{1}=v_{B}=v_{E}=v_{F}$

The kinematic velocity magnitude of the tunnel face can be written as follows:

$$
v_{T}=\sqrt{2} v_{1} / 2
$$

Therefore, the vertical velocity magnitude of the centroid of zones I, II, and III can be separately expressed as follows:

$$
v_{I, c}=\frac{\sqrt{2}}{2} v_{1} ; \quad v_{I I, c}=v_{1} ; \quad v_{I I I, c}=\frac{\sqrt{2}}{2} v_{1}
$$

where $v_{I, c}, v_{I I, c}$, and $v_{I I I, c}$ are respectively the vertical velocity of the centroid of zones I, II, 
and III. In Figure 6, the velocity discontinuity surfaces consist of line $\overline{A B}$, line $\overline{O F}$ and the circular arc BE.

\section{Criterion of the limit collapse thickness}

In this study, there are two possible failure patterns in front of the tunnel face, as shown in Figure 4. The shapes of the failure mechanism depend on the ratio $C / D$. When $C / D$ is 0.5 , the failure mechanism consists of three zones, i.e., zones I, II and III. When the values of $C / D$ are higher than 0.5, zone IV (i.e., the trapezoid $E F J K$ with height $H$ ) may be the possible failure zone, which is subjected to the influence of the vertical soil arching effect, as shown in Figure 8. The shape of the fracture mechanism is consistent with that of a test obtained by Schofield (1980).

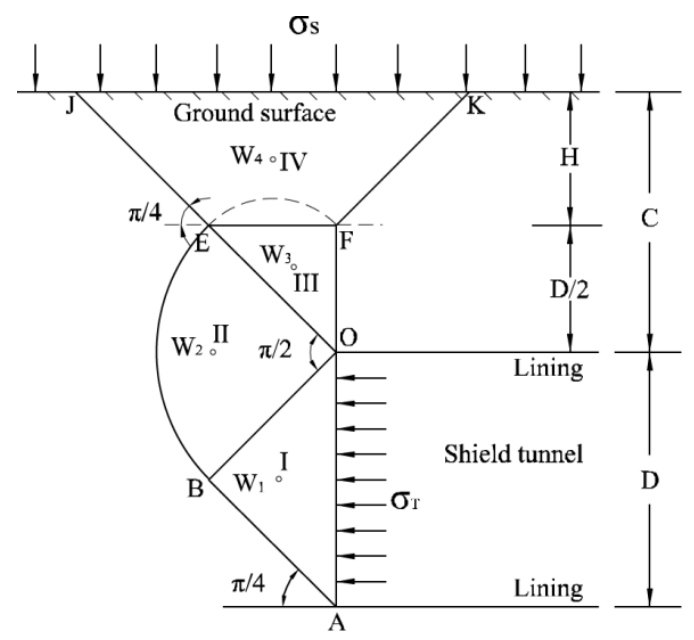

Fig. 8 Assumed failure mechanism when $C / D>0.5$

In Figure 8, the weight of the soils in trapezoid EFJK, the soil undrained cohesion $c_{u}$ and the uniform surcharge loading $\sigma_{s}$ on the ground surface play an important role in evaluating the stability of the soil mass in trapezoid EFJK. When the soil mass in trapezoid EFJK (i.e., zone IV) is unstable, zone IV becomes a possible fracture zone. In this case, the criterion of the limit collapse thickness needs to be defined to obtain the critical collapse pressure. Assuming that the collapse of the tunnel face just reaches the ground surface, the soils in zone IV can be regarded as loose earth, and the collapse pressure acting on line $\overline{E F}$ can be obtained using the Terzaghi's theory of relative soil pressure, which is modified as shown in Figure 9. 


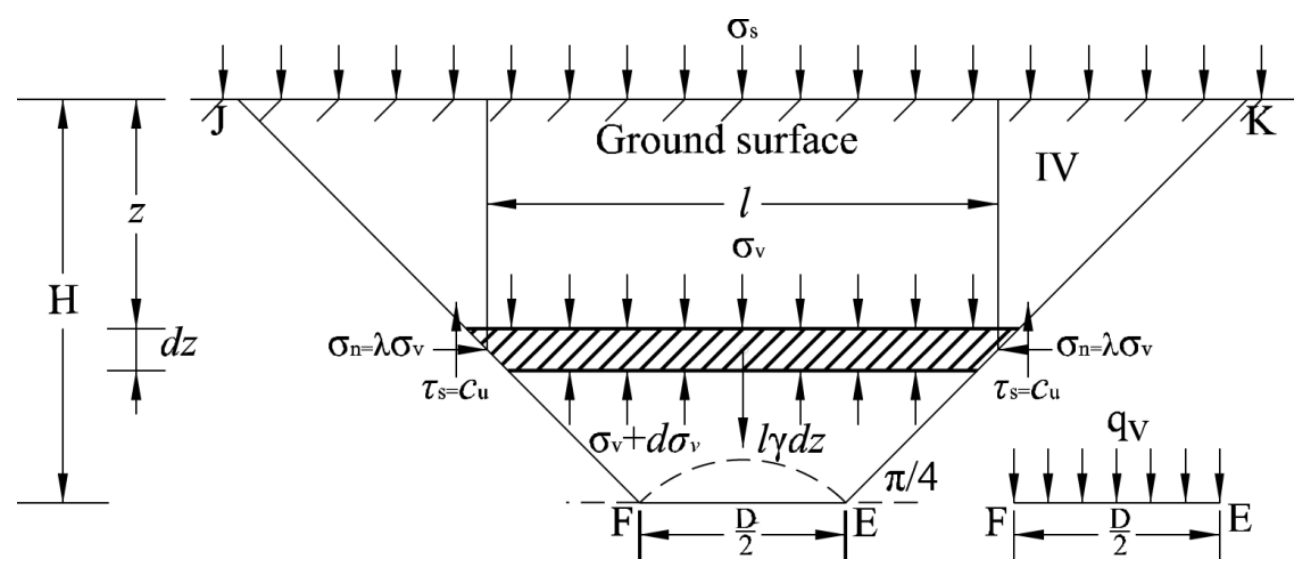

Fig. 9 Modified Terzaghi's theory of relative soil pressure

The contribution to the collapse pressure caused by the weight of the soils in trapezoid EFJK, uniform surcharge loading $\sigma_{s}$ on the ground surface and the soil undrained cohesion $c_{u}$ can be equivalent to a uniformly distributed load pressure $q_{V}$ acting on line $\overline{E F}$ and expressed as follows:

$q_{v}=\gamma H+\sigma_{s}+c_{u} \ln \left(\frac{D}{D+4 H}\right)(5)$

where $H$ is the possible collapse thickness, $\gamma$ is the soil unit weight, $D$ is the diameter of the circular tunnel, and $c_{u}$ is the soil undrained cohesion.

As $H=C-\frac{D}{2}$ in Figure 8, equation (5) becomes equation (6), as shown below:

$$
q_{v}=\gamma D\left(\frac{C}{D}-\frac{1}{2}\right)-c_{u} \ln \left(\frac{4 C}{D}-1\right)+\sigma_{s}(6)
$$

where $C$ is the depth of cover. Equation (6) is the criterion of the limit collapse thickness.

\section{Analysis of the limit collapse thickness}

\section{No uniform surcharge loading $\sigma_{s}$ on the ground surface}

Neglecting the uniform surcharge loading, equation (6) is simplified as follows:

$$
q_{v}=\gamma D\left(\frac{C}{D}-\frac{1}{2}\right)-c_{u} \ln \left(\frac{4 C}{D}-1\right)(7)
$$

When $q_{V} \leq 0$, that is, $\frac{\gamma D}{c_{u}} \leq \ln \left(4 C / D^{-1}\right) /\left(C / D^{-1 / 2}\right)$, the soil mass in trapezoid EFJK is stable. Therefore, the fracture areas in front of the tunnel face involve zones I, II and III, and the 
limit collapse thickness is $0.5 D$; otherwise, it is the depth of cover, $C(\mathrm{H}+\mathrm{D} / 2)$. An intuitive design chart to evaluate the limit collapse thickness is shown in Figure 10.

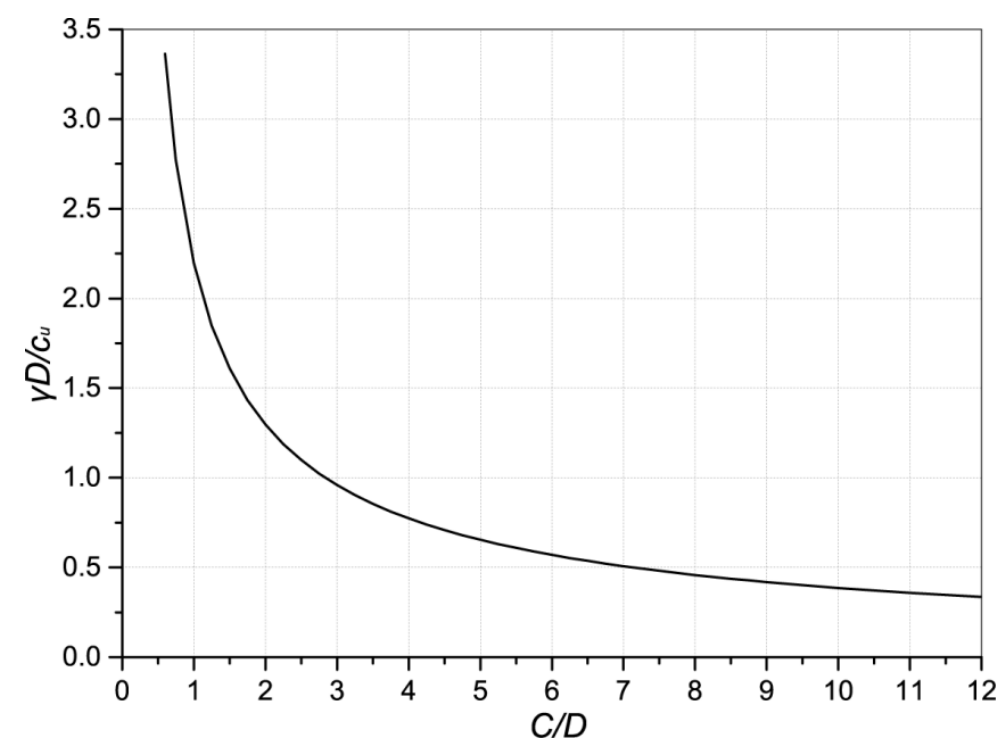

Fig. 10 Design chart for the relationship between $\gamma D / c_{u}$ and $C / D$

For a certain value of $C / D$, it can be seen from Figure 10 that the soil mass in trapezoid $E F J K$ is stable when the value of $\gamma D / c_{u}$ is under or on the curve. Therefore, the limit collapse thickness is $0.5 \mathrm{D}$; otherwise, it is the depth of cover, $C(\mathrm{H}+\mathrm{D} / 2)$.

\section{Uniform surcharge loading $\sigma_{s}$ on the ground surface}

When considering uniform surcharge loading for $q_{V} \leq 0$, that is, $\gamma D\left(\frac{C}{D}-\frac{1}{2}\right)-c_{u} \ln \left(\frac{4 C}{D}-1\right)+\sigma_{s} \leq 0$, the soil mass in trapezoid $E F J K$ is stable. Therefore, the fracture areas in front of the tunnel face involve zones I, II and III, and the limit collapse thickness is $0.5 D$; otherwise, it is the depth of cover, $C(\mathrm{H}+\mathrm{D} / 2)$. A design chart is necessary to more intuitively evaluate the limit collapse thickness, as shown in Figure 11. 


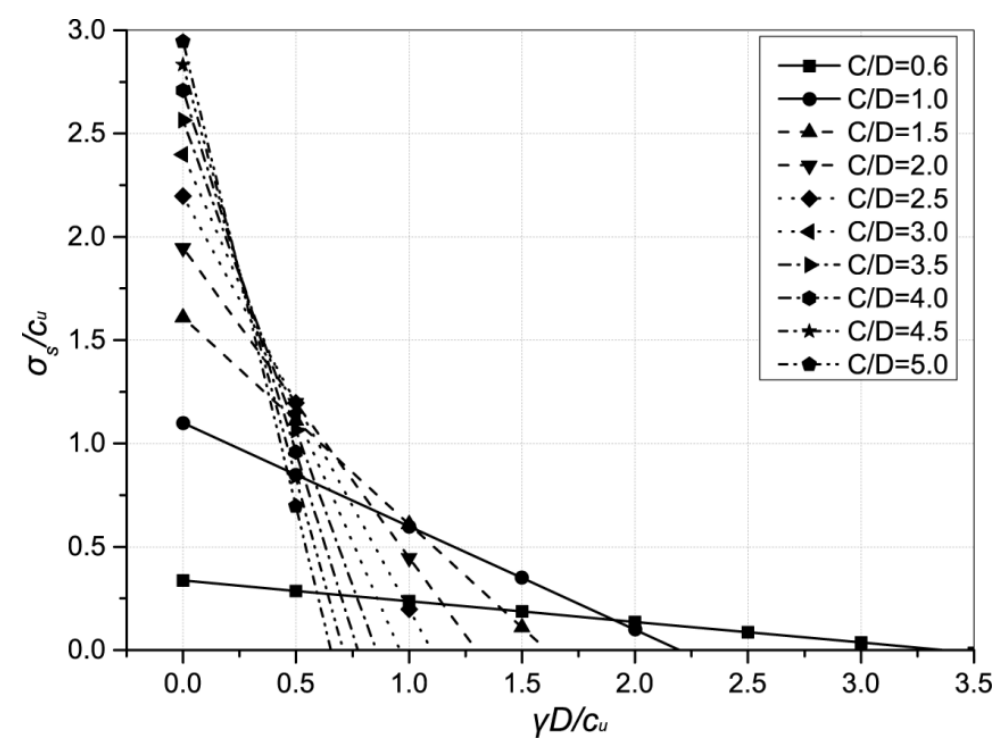

Fig. 11 Design chart for the relationship between $\gamma D / c_{u}$ and $\sigma_{s} / c_{u}$

For a certain value of $C / D$, Figure 11 shows that the soil mass in trapezoid $E F J K$ is stable, and the contribution of the uniform surcharge loading $\sigma_{s}$ to the critical collapse pressure must be considered when the point with coordinates $\left(\gamma D / c_{u}, \sigma_{S} / c_{u}\right)$ is under or on the curve generated from equation (6). Therefore, the limit collapse thickness is equal to $0.5 \mathrm{D}$; otherwise, it is equal to $C(\mathrm{H}+\mathrm{D} / 2)$.

\section{Critical collapse pressure}

This paper aims at finding a more accurate critical collapse pressure of a circular tunnel driven by a shield in purely cohesive soils. Based on the kinematic approach of the limit analysis, together with the slip line theory a multi-zone failure mechanism is proposed. As described in the above section, the shape of the failure mechanism depends on the values of $C / D$. As the critical collapse pressure depends on the failure mechanism, the critical collapse pressure of the tunnel face is calculated as follows.

\section{C/D equal to 0.5}

Figure 12 shows the fracture mechanism when $\mathrm{C} / \mathrm{D}$ is 0.5 . 


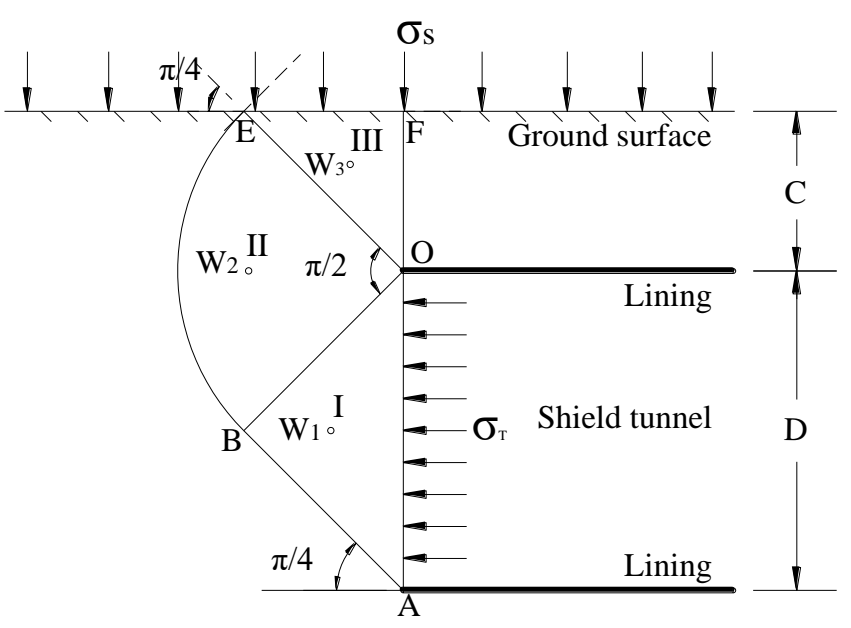

Fig. 12 Failure mechanism for $C / D=0.5$

This fracture mechanism consists of three zones. To obtain the critical collapse pressure using the limit analysis method, the rate of external work and that of the internal energy dissipation must be determined. The rate of external work is caused by the weight of the three zones, the possible uniform surcharge loading on the ground surface and the collapse pressure on the tunnel face. The rate of the internal energy dissipation is determined along the different velocity discontinuity surfaces. According to the analysis of the admissible continuous velocity field (see Figure 6), the rate of external work and that of the internal energy dissipation are calculated as follows.

The rate of work of the weight of the three zones, i.e., zone I, II and III, is shown below:

$$
\dot{p}_{\gamma}=\iiint_{V} \gamma \cdot v d V=\gamma \sum_{i=1}^{3} v_{i} V_{i}=\frac{\pi+6 \sqrt{2}}{32} \gamma D^{2} v_{1}
$$

The rate of work of the collapse pressure of the tunnel face is shown below:

$$
\dot{p}_{\sigma_{T}}=\iint_{A_{T}} \sigma_{T} \cdot v_{n}^{T} d A=-\sqrt{2} \sigma_{T} D v_{1} / 2(9)
$$

As undrained behaviour is assumed, the soil deforms at constant volume, as shown below.

$$
\iint_{A_{s}} v_{n}^{s} d A=\iint_{A_{T}} v_{n}^{T} d A
$$

Thus, the rate of work of possible uniform surcharge loading on the ground surface is shown below:

$$
\dot{p}_{\sigma_{S}}=\iint_{A_{s}} \sigma_{S} \cdot v_{n}^{s} d A=\iint_{A_{T}} \sigma_{s} v_{n}^{T} d A=\sqrt{2} \sigma_{s} D v_{1} / 2
$$

where $V_{i}$ is the volume of region $i, v_{i}$ is the vertical velocity of centroid $i$ in the volume of 
region $i(i=1,2,3), v_{n}^{s}$ is the downwards normal velocity at the ground surface, and $v_{n}^{T}$ is the outward normal velocity on the tunnel face. $A_{T}$ and $A_{s}$ are the deforming areas on the tunnel face and at the ground surface, respectively.

The rate of internal energy dissipation along the different velocity discontinuity surfaces is shown below:

$$
\dot{D}_{S_{i}}=\iint_{S} c_{u} \cdot v d S=c_{u} \cdot \sum_{i=1}^{n} v_{i} S_{i}
$$

where $S_{i}$ is the lateral surface along line $\overline{A B}$, line $\overline{O F}$ and arc $B E$. The rate of the internal energy dissipation along the discontinuous surface is given as follows:

$$
\dot{D}_{S_{i}}=\frac{3 \sqrt{2}+\sqrt{2} \pi}{4} c_{u} D v_{1}
$$

The work equation consists of equating the rate of work of the external forces to the rate of internal energy dissipation and is given as follows:

$$
c_{u} \cdot \sum_{i=1}^{n} v_{i} S_{i}=\gamma \sum_{i=1}^{n} v_{i} V_{i}+\iint_{A_{s}} \sigma_{S} \cdot v_{n}^{s} d A+\iint_{A_{T}} \sigma_{T} \cdot v_{n}^{T} d A(14)
$$

After simplification, the tunnel collapse pressure can be written as follows:

$$
\sigma_{T}=\gamma D N_{\gamma}-c_{u} N_{c}+\sigma_{s} N_{s}(15)
$$

where $N_{\gamma}, N_{c}$, and $N_{s}$ are non-dimensional coefficients that represent, respectively, the effect of the soil weight, the cohesion, and the surcharge loading. The expressions of these coefficients are given as follows:

$$
\begin{aligned}
& N_{\gamma}=\frac{12+\sqrt{2} \pi}{32} \\
& N_{c}=\frac{3+\pi}{2} \\
& N_{s}=1
\end{aligned}
$$

Substituting equations (16), (17) and (18) into equation (15), the critical collapse pressure becomes as follows: 


$$
\sigma_{T}=0.5138 \gamma D-3.0708 c_{u}+\sigma_{s}(19)
$$

When $\sigma_{T}=0$, equation (19) becomes as follows:

$$
\left[\sigma_{s}+0.5138 \gamma D\right] / c_{u}=3.0708
$$

\section{C/D greater than 0.5}

Figure 13 shows the fracture mechanism when C/D is greater than 0.5 .

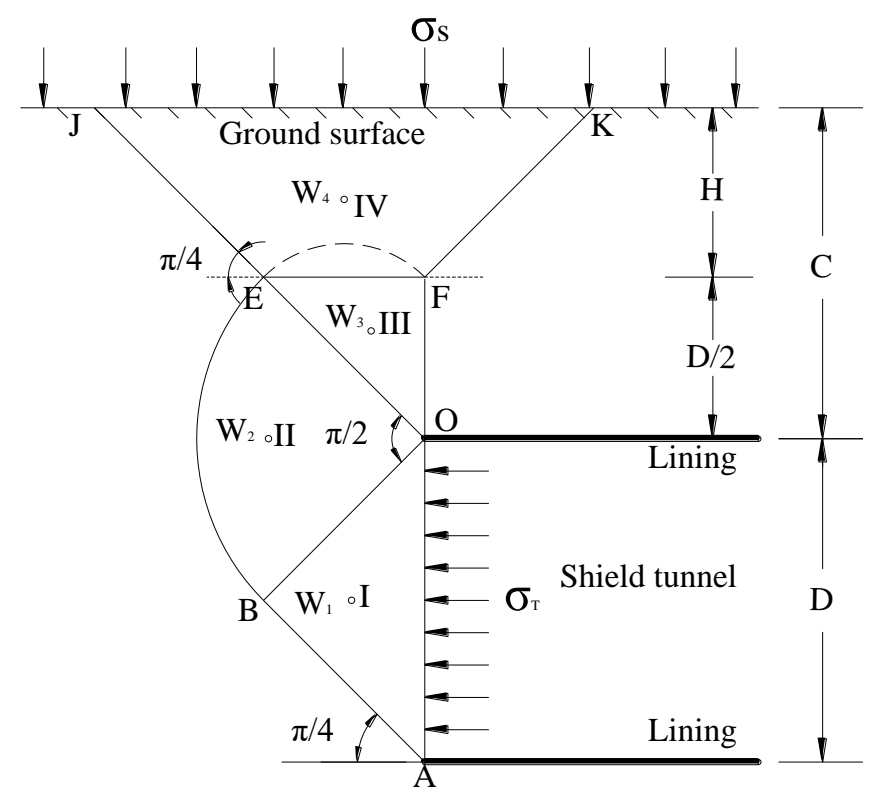

Fig. 13 Failure mechanism for $C / D>0.5$

This fracture mechanism involves four zones. To obtain the critical collapse pressure of the tunnel face, the rate of work of the weight of the four regions, the possible uniform surcharge loading on the ground surface and the collapse pressure on the tunnel face must be determined. In addition, the rate of internal energy dissipation along the different velocity discontinuity surfaces must be obtained. According to the analysis of the admissible continuous velocity field (see Figure 6), the rate of external work and that of the internal energy dissipation are given as follows.

The equation for the rate of work of the weight of the three zones, i.e., zones I, II and III, is the same as in equation (8). The contributions of the weight of the soils in zone IV, the uniform surcharge loading $\sigma_{s}$ on the ground surface and the soil undrained cohesion $c_{u}$ to the critical collapse pressure are equivalent to that of the equivalent uniform distribution loads $q_{V}$ acting on line $\overline{E F}$. Therefore, the rate of work of the weight of the soils in zone IV, the possible uniform surcharge 
loading $\sigma_{s}$ and the soil undrained cohesion $c_{u}$ can be expressed as follows:

$$
\dot{p}_{q_{v}}=\iint_{A_{q}} q_{v} \cdot v_{n}^{q} d A=\frac{\sqrt{2}}{2}\left[\gamma D\left(\frac{C}{D}-\frac{1}{2}\right)-c_{u} \ln \left(4 \frac{C}{D}-1\right)+\sigma_{s}\right] D v_{1}(21)
$$

where $v_{n}^{q}$ is the downwards normal velocity of the equivalent uniform distribution loads $q_{V}$ acting on line $\overline{E F}$, and $A_{q}$ is equal to the length of line $\overline{E F}$ multiplied by unit thickness.

The equation for the rate of work of the collapse pressure of the tunnel face is the same as in equation (9).

The equation for the rate of internal energy dissipation along the different velocity discontinuity surfaces is the same as in equation (13). The work equation is obtained by equating the rate of work of the external forces to the rate of internal energy dissipation as follows:

$$
c_{u} \cdot \sum_{i=1}^{n} v_{i} S_{i}=\gamma \sum_{i=1}^{n} v_{i} V_{i}+\iint_{A_{n}} q_{\nu} v d A^{\prime}+\iint_{A_{T}} \sigma_{T} \cdot v_{n}^{T} d A_{(22)}
$$

After simplification, it is found that the tunnel collapse pressure is given by

$$
\sigma_{T}=\gamma D N_{\gamma}-c_{u} N_{c}+\sigma_{s} N_{s}
$$

where $N_{\gamma}, N_{c}$ and $N_{s}$ are non-dimensional coefficients that represent the effect of the soil weight, the cohesion, and the surcharge loading, respectively. The expressions of these coefficients are given as follows:

$$
\begin{aligned}
& N_{\gamma}=N_{\gamma}^{\prime}+N_{\gamma}^{\prime \prime}=\frac{12+\sqrt{2} \pi}{32}+\left(\frac{C}{D}-\frac{1}{2}\right)(24) \\
& N_{c}=N_{c}^{\prime}+N_{c}^{\prime \prime}=\frac{3+\pi}{2}+\ln \left(\frac{4 C}{D}-1\right)(25) \\
& N_{s}=1(26)
\end{aligned}
$$

where $N_{\gamma}^{\prime}=\frac{12+\sqrt{2} \pi}{32}$ is the effect of the soil weight of regions I, II and III; $N_{\gamma}^{\prime \prime}=\left(\frac{C}{D}-\frac{1}{2}\right)$ is the possible effect of the soil weight of zone IV; $N_{c}^{\prime}=\frac{3+\pi}{2}$ is the effect of cohesion along line $\overline{A B}$, line $\overline{O F}$ and arc $B E ; \quad N_{c}^{\prime \prime}=\ln \left(\frac{4 C}{D}-1\right)$ is the possible effect of cohesion of the soils in 
zone IV; and $N_{s}=1$ is the effect of a possible uniform surcharge loading on the ground surface.

Note that in equations (24), (25) and (26), whether to consider the values of $N_{\gamma}^{\prime \prime}, N_{c}^{\prime \prime}$ and $N_{s}$ depends on the dimensionless parameters $\gamma D / c_{u}, \sigma_{S} / c_{u}$ and $C / D$. Thus, according to equation (6), the critical collapse pressure as a function of $C / D$ is calculated as follows:

(a) $\frac{\gamma D}{c_{u}}\left(\frac{C}{D}-\frac{1}{2}\right)+\frac{\sigma_{s}}{c_{u}}-\ln \left(\frac{4 C}{D}-1\right) \leq 0$

Under this condition, $N_{\gamma}^{\prime \prime}$ and $N_{c}^{\prime \prime}$ are both equal to zero. Thus, equations (24) and (25) are reduced, respectively, to the following:

$$
\begin{aligned}
& N_{\gamma}=\frac{12+\sqrt{2} \pi}{32}(27) \\
& N_{c}=\frac{3+\pi}{2}(28)
\end{aligned}
$$

In this case, the equivalent uniform distribution load $q_{V}$ is less than or equal to zero. Thus, equation (26) becomes as follows:

$$
N_{s}=0(29)
$$

Substituting equations (27), (28) and (29) into equation (23), the critical collapse pressure is calculated as follows:

$\sigma_{T}=0.5138 \gamma D-3.0708 c_{u}(30)$

When $\sigma_{T}=0$, equation (30) becomes as follows:

$$
\begin{aligned}
& \frac{\gamma D}{c_{u}}=5.977(31) \\
& \text { (b) } \frac{\gamma D}{c_{u}}\left(\frac{C}{D}-\frac{1}{2}\right)+\frac{\sigma_{s}}{c_{u}}-\ln \left(\frac{4 C}{D}-1\right)>0
\end{aligned}
$$

In this case, $N_{\gamma}^{\prime \prime}, N_{c}^{\prime \prime}$ and $N_{s}$ must be considered. Substituting equations (24), (25) and (26) into equation (23), the critical collapse pressure is calculated as follows: 
$\sigma_{T}=\left[0.5138+\left(\frac{C}{D}-\frac{1}{2}\right)\right] \gamma D-\left[3.0708+\ln \left(\frac{4 C}{D}-1\right)\right] c_{u}+\sigma_{s}(32)$

When $\sigma_{T}=0$, equation (32) becomes as follows:

$\left[\sigma_{s}+\left(0.0138+\frac{C}{D}\right) \gamma D\right] / c_{u}=3.0708+\ln \left(\frac{4 C}{D}-1\right)$

\section{Analytical comparisons}

In this study, it is assumed that the undrained shear strength $c_{u}$ of a purely cohesive soil is constant with depth. Thus, six variables model of the plane strain heading is formed by the following set: $\left\{\sigma_{\mathrm{T}}, \sigma_{s}, C, D, c_{u}, \gamma\right\}$. Dimensional analysis is used to solve the problem via the following dimensionless sets: $\left\{\sigma_{\mathrm{T}} / c_{u}, \sigma_{s} / c_{u}, C / D, \gamma D / c_{u}\right\}$. As undrained behaviour is assumed, the first two in the dimensionless set are replaced by a single parameter, $\left(\sigma_{s}-\sigma_{T}\right) / c_{u}$, which becomes the only variable for limiting stability once the values of the independent parameters $C / D$ and $\gamma D / c_{u}$ have been fixed.

As the present model in this paper is $2 \mathrm{D}$, the existing classical solutions of two-dimension models or some recent three-dimension models, including analytical and numerical solutions, are chosen to validate the results of the present model. For the cohesive soils, a traditional approach is to evaluate stability of a circular tunnel face in terms of a "load factor", usually denoted as $N$ and defined as follows:

$$
N=\left[\sigma_{s}-\sigma_{T}+\gamma D \cdot(C / D+1 / 2)\right] / c_{u}
$$

The advantage of using the load factor to assess stability can reduce the complexity of the final results. However, several researchers demonstrated that the values of $N$ are dependent on the values of $\gamma D / c_{u}$. Therefore, the analytical comparisons are made under different values of $\gamma D / c_{u}$, as follows. 
(a) $\gamma D / c_{u}=0$

In this case, equation (34) reduces to the following:

$$
N=\left(\sigma_{s}-\sigma_{T}\right) / c_{u}
$$

In this study, the load factor can be expressed as follows:

$$
N=N_{c}=(3+\pi) / 2+\ln (4 C / D-1)
$$

The analytical comparisons of the load factor of the present model and the existing solutions are shown in Figure 14.

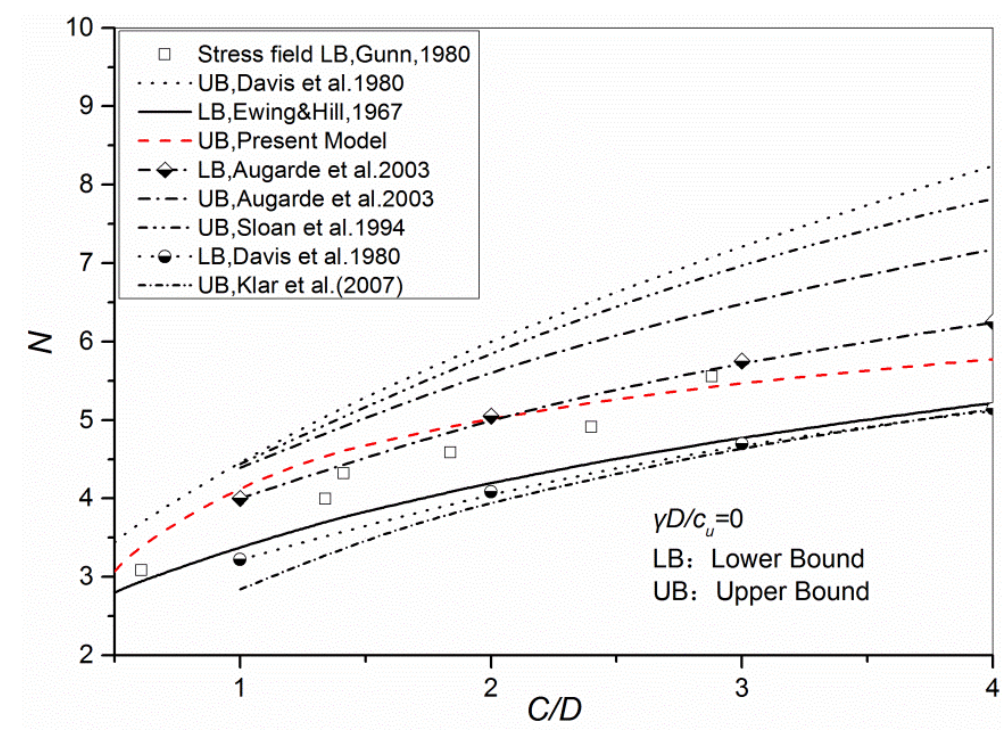

Fig. 14 Analytical comparisons of $N$ of the present model and the existing solutions

Figure 14 shows that the reasonable solutions of the problem should be between the upper-bound solutions proposed by Davis et al.(1980) and the lower-bound solutions proposed by Davis et al.(1980). In Figure 14, the upper-bound solutions proposed by Sloan et al.(1994) and by Augarde et al.(2003) are numerical solutions obtained using finite element limit analysis methods based on the improved rigid-blocks mechanism proposed by Davis et al.(1980). The upper-bound solutions proposed by Augarde et al.(2003) were derived from updating the upper-bound solutions proposed by Sloan et al.(1994) using advanced algorithms of the finite element limit analysis. The upperbound solutions proposed by Augarde et al.(2003) are more accurate bounds on stability parameters. 
Therefore, the range of best solutions of the problem should be reduced to between the upper-bound solutions proposed by Augarde et al.(2003) and the lower-bound solutions proposed by Davis et al(1980). The comparisons of the upper-bound solutions proposed by Davis et al.(1980), Sloan et al.(1994) and Augarde et al.(2003) show that the best upper-bound solutions more closely approximate the lower-bound solutions. In the lower-bound solutions shown in Figure 14, the three lower-bound solutions proposed by Davis et al.(1980), Augarde et al.(2003), Ewing and Hill(1967) and the present model are calculated based on smooth linings. Only the lower-bound solution proposed by Gunn(1980) is computed based on rough linings. The lower-bound solutions based on rough linings would be appropriate in a purely cohesive soil. Thus, the reasonable upper-bound solutions more closely approximate the lower-bound solutions proposed by Gunn(1980). It can be seen from Figure 14 that the upper-bound solutions proposed by the present model agree well with the lower-bound solutions proposed by Gunn(1980) when $C / D<4$. The upper-bound solutions obtained by the present model are better than other upper-bound solutions. The present upper bound mechanism more closely approximates the failure mechanism in the actual project and a safe estimate of the tunnel pressure.

(b) $\gamma D / c_{u}>0$

For the more general case of a cohesive soil, the traditional method used to assess the critical collapse pressure is using the following equation

$$
\sigma_{T}=\gamma \cdot D \cdot N_{\gamma}-c_{u} \cdot N_{c}+\sigma_{s} \cdot N_{s}
$$

Since the critical coefficient $N_{s}$ is equal to 1.0 for purely cohesive soils under undrained conditions, equation (37) becomes

$$
\sigma_{T}-\sigma_{s}=\gamma \cdot D \cdot N_{\gamma}-c_{u} \cdot N_{c}
$$

The equation (38) can be transformed into

$$
\left[\left(\sigma_{s}-\sigma_{T}\right)+\gamma \cdot D \cdot N_{\gamma}\right] / c_{u}=N_{c}
$$


Adding $\gamma \cdot D \cdot\left(C / D+1 / 2-N_{\gamma}\right) / c_{u}$ on both sides of the equations (39), the equation (39) becomes

$$
\left[\left(\sigma_{s}-\sigma_{T}\right)+\gamma \cdot D \cdot(C / D+1 / 2)\right] / c_{u}=N_{c}+\gamma \cdot D \cdot\left[(C / D+1 / 2)-N_{\gamma}\right] / c_{u}
$$

Substituting equation (34) into equation (40), the load factor can be expressed as

$$
N=N_{c}+\gamma \cdot D \cdot\left[(C / D+1 / 2)-N_{\gamma}\right] / c_{u}
$$

The equation (41) shows that the load factor $N$ is dependent of $\gamma D / c_{u}$ when considering the effect of the self weight of the soil.

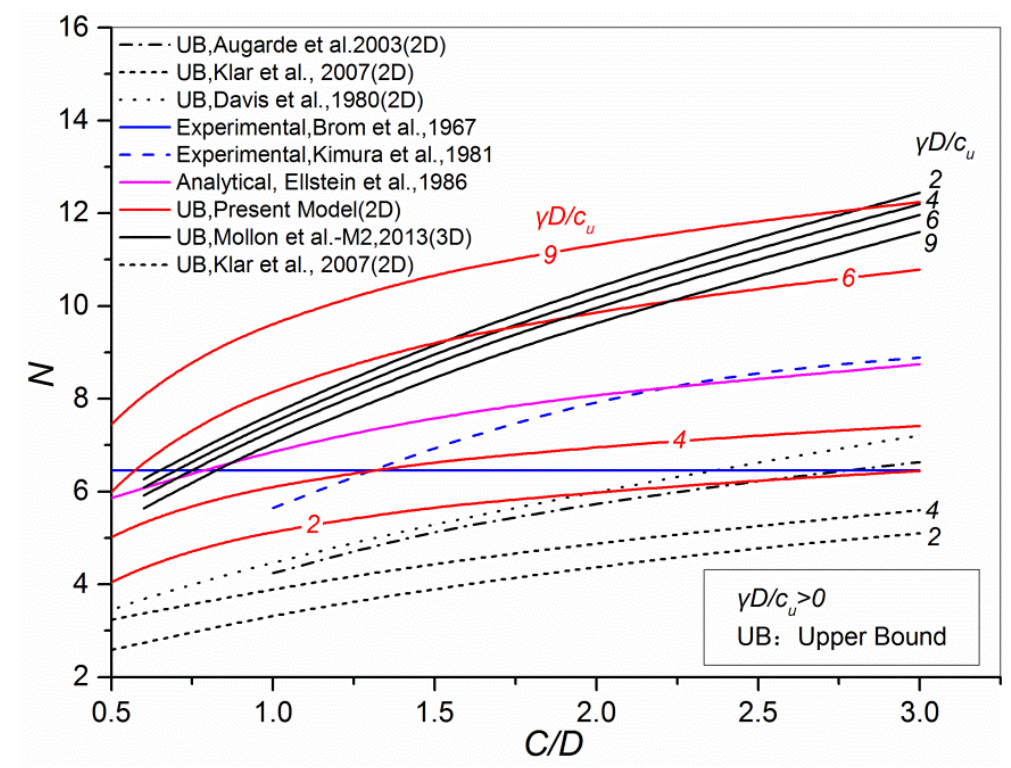

Fig. 15 Analytical comparisons of $\mathbf{N}$ between the present model and the existing solutions

$$
\left(\gamma D / c_{u}>0\right)
$$

Figure 15 shows that the upper-bound solutions proposed by Davis et al.(1980), Augarde et al. (2003) and Klar et al.(2007) are lower than the experimental and analytical solutions obtained by Brom et al.(1967), Kimura et al.(1981) and Ellstein et al.(1986) for values of C/D from 0.5 to 3.0 when $\gamma D / c_{u}>0$. This illustrates that the upper-bound solutions proposed by Davis et al.(1980), Augarde et al. (2003) and Klar et al.(2007) should be safe and conservative. For the present model, the values of $\mathrm{N}$ corresponding to certain value of $\mathrm{C} / \mathrm{D}$ increase with increasing the values of $\gamma D / c_{u}$.

When the values of $\gamma D / c_{u}$ is lower than 2.0, the upper-bound solutions proposed by the present model is safe and conservative. When the values of $\gamma D / c_{u}$ is greater than 6.0, the upper-bound solutions is unsafe. Therefore, the upper-bound solutions proposed by the present model may be the best solutions for the value of $\gamma D / c_{u}$ equal to 5.0. The upper-bound solutions proposed by Mollon 
et al.(2013) are greater than these experimental and analytical solutions. It shows that the critical collapse pressures obtained by Mollon et al.(2013) should be smaller than the real solutions required to ensure the safe of the tunnel face. Meanwhile, the values of $\mathrm{N}$ corresponding to certain value of $\mathrm{C} / \mathrm{D}$ decrease with increasing the values of $\gamma D / c_{u}$. This is in conflict with the real situation in the actual project.

Based on the research data proposed by Kimura et al.(1981), Ellstein et al.(1986), Augrade et al.(2003), Klar et al.(2007), Mollon et al.(2013) and the present model, the fitting equations of the critical coefficients $N_{c}$ versus the ratio of the cover to the depth (C/D) can be expressed as

$$
N_{c}=A \sqcap \ln (4 C / D-1)+B
$$

Where A and B are constant coefficients which are showed in Table 1.

Table 1 Constant coefficients and coefficients of determination of fitting equations

\begin{tabular}{llll}
\hline models & $\mathrm{A}$ & $\mathrm{B}$ & $\mathrm{R}^{2}$ \\
\hline Present model & 1.0 & 3.0708 & 1.0 \\
\hline $\begin{array}{l}\text { Model proposed by } \\
\text { Kirmura et al.(1981) }\end{array}$ & 2.5719 & 0.9647 & \\
\hline $\begin{array}{l}\text { Model proposed by } \\
\text { Augarde et al.(2003) }\end{array}$ & 1.9717 & 5.9766 & 0.9925 \\
\hline $\begin{array}{l}\text { Model proposed by } \\
\text { Ellstein et al.(1986) }\end{array}$ & 1.2071 & 1.626 & 0.9904 \\
\hline $\begin{array}{l}\text { Model proposed by } \\
\text { Klar et al.(2007) }\end{array}$ & & 0.9696 \\
\hline $\begin{array}{l}\text { Model proposed by } \\
\text { Mollon et al.(2013) }\end{array}$ & 4.0487 & 0.96867 \\
\hline
\end{tabular}

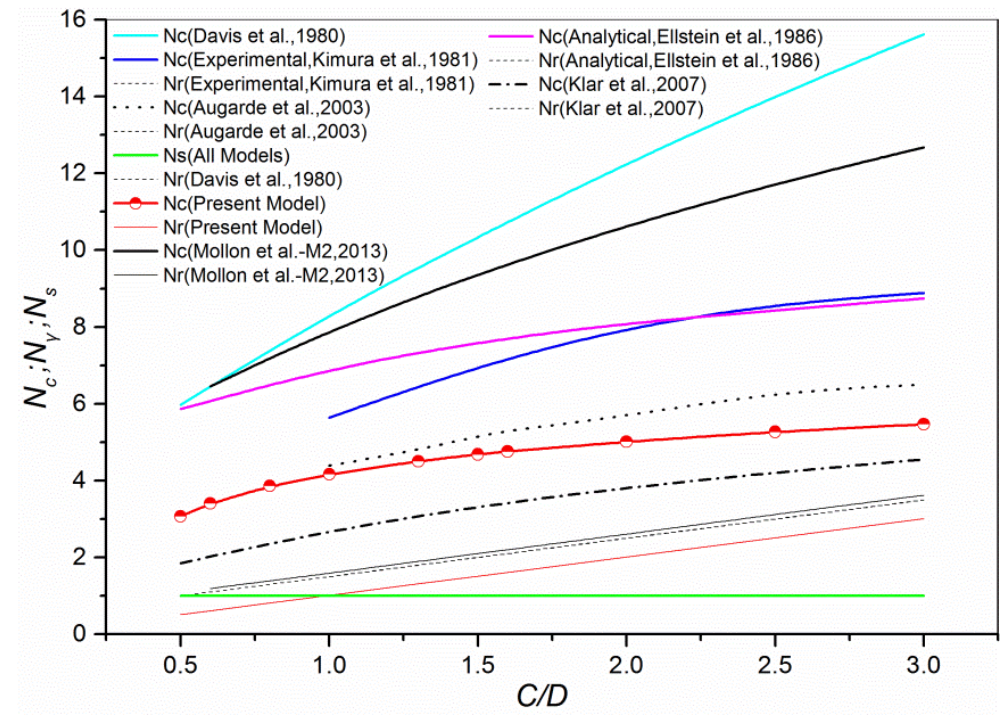

(a) $N_{c}-N_{\gamma}-N_{s}$ 


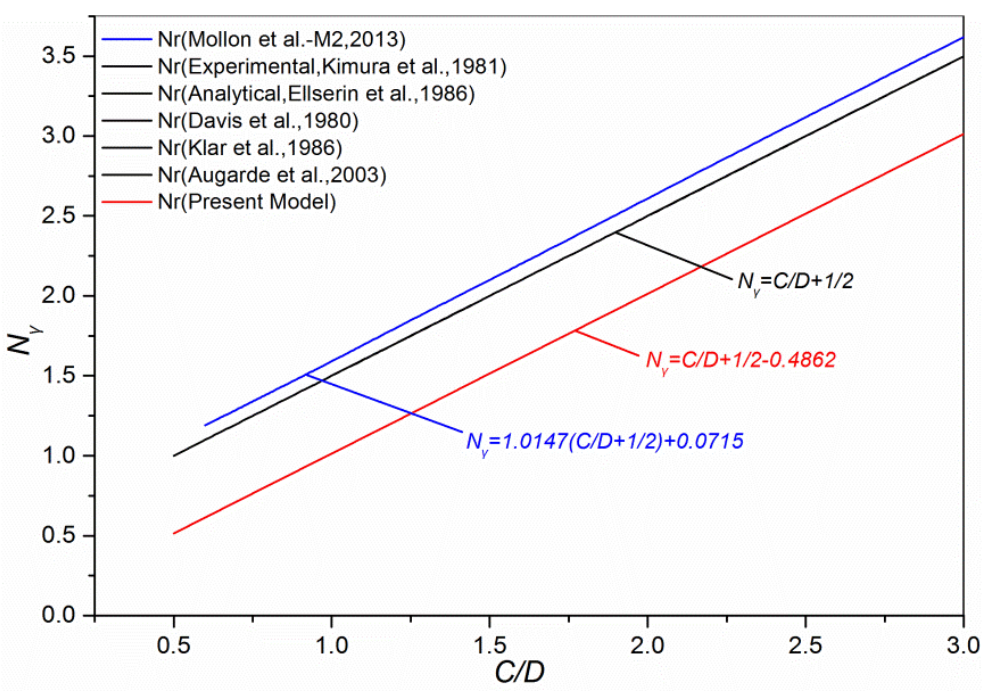

(b) $N_{\gamma}$

Fig. 16 Comparison of $N_{c}, N_{\gamma}$ and $N_{s}$ of the existing models and the present model:

(a) $N_{c^{-}}-N_{\gamma}-N_{s} ;$ (b) $N_{r}$

Figure 16 illustrates the comparisons of critical coefficients, i.e., $N_{c}, N_{\gamma}$ and $N_{s}$, of the existing models and the present model. In Figure 16, the critical coefficient $N_{s}$ (i.e., the effect of surcharge loading) of all models is equal to 1.0 , which shows that the effect of surcharge loading to the critical collapse pressure does not vary with the values of $C / D$. The reason is that since these methods for cohesive material involves no volume change then the decrease in area of the tunnel must equal the area of ground loss at the surface. It can be seen from Figure 16(a) that the values of $N_{c}$ is greater than the values of $N_{\gamma}$ when $0.5 \leq C / D \leq 3.0$, which indicates that the effect of cohesion to the critical collapse pressure is more remarkable than that of the soil weight. Figure 16(b) shows that the critical coefficient $N_{\gamma}$ of all models linearly increases with increasing the values of $C / D$. The critical coefficient $N_{\gamma}$ of these models, except for the present model and the M2 model proposed by Mollon et al.(2013), is fixed to equal to $(C / D+1 / 2)$. It can be seen from Figure 16(b) that the increase of critical coefficient $N_{\gamma}$ with respect to $N_{\gamma}=(C / D+1 / 2)$ by the M2 model proposed by Mollon et al.(2013) is smaller than $7.15 \%$ when $C / D \geq 0.5$. However, the maximum decrease of critical coefficient $N_{\gamma}$ with respect to $N_{\gamma}=(C / D+1 / 2)$ by the present model can attain more than $48 \%$ 
when $C / D \geq 0.5$. Hence it is reasonable to assume that the load factor $N$ depends on the values of $\gamma D / c_{u}$ when using a kinematically permissible mechanism.

\section{Case analysis}

In this study, the diameter $\mathrm{D}$ of the circular tunnel in purely cohesive soil is equal to $10 \mathrm{~m}$, a unit weight $\gamma$ of the soils is $18 \mathrm{kN} / \mathrm{m}^{3}$, the undrained cohesions $c_{u}$ of the soils are $20 \mathrm{kPa}$ and $30 \mathrm{kPa}$, respectively. Meanwhile, it is assumed that the uniform surcharge loading $\sigma_{s}$ on the ground surface does not exist. Since the critical collapse pressure depends on the fracture mechanism which depends on the values of $\mathrm{C} / \mathrm{D}$ and $\gamma D / c_{u}$, the suitable equations to calculate the critical collapse pressure need to be chose. When $\mathrm{C} / \mathrm{D}=0.5$, the critical collapse pressures $\sigma_{T}$ obtained using equation (19) with $\sigma_{S}=0$ are equal to $31.07 \mathrm{kPa}$ and $0.36 \mathrm{kPa}$ for the two $c_{u}$ values $(20 \mathrm{kPa}$ and $30 \mathrm{kPa}$ ). When the values of $\mathrm{C} / \mathrm{D}$ are greater than 0.5 , the equivalent uniform distribution loads $q_{V}$ calculated using equation (7) are greater than zero for the two $c_{u}$ values. Hence, the critical collapse pressure $\sigma_{T}$ can be calculated using equation (32) with $\sigma_{S}=0$.

In this study, the solutions of the critical collapse pressure obtained by Davis et al. (1980), Augarde et al.(2003), Klar et al. (2007), Mollon et al. (2013), and the present approach are given in Figs. 17(a) and (b). In addition to the numerical solution, note that the rest of these results are based on the kinematical approach of the limit analysis.

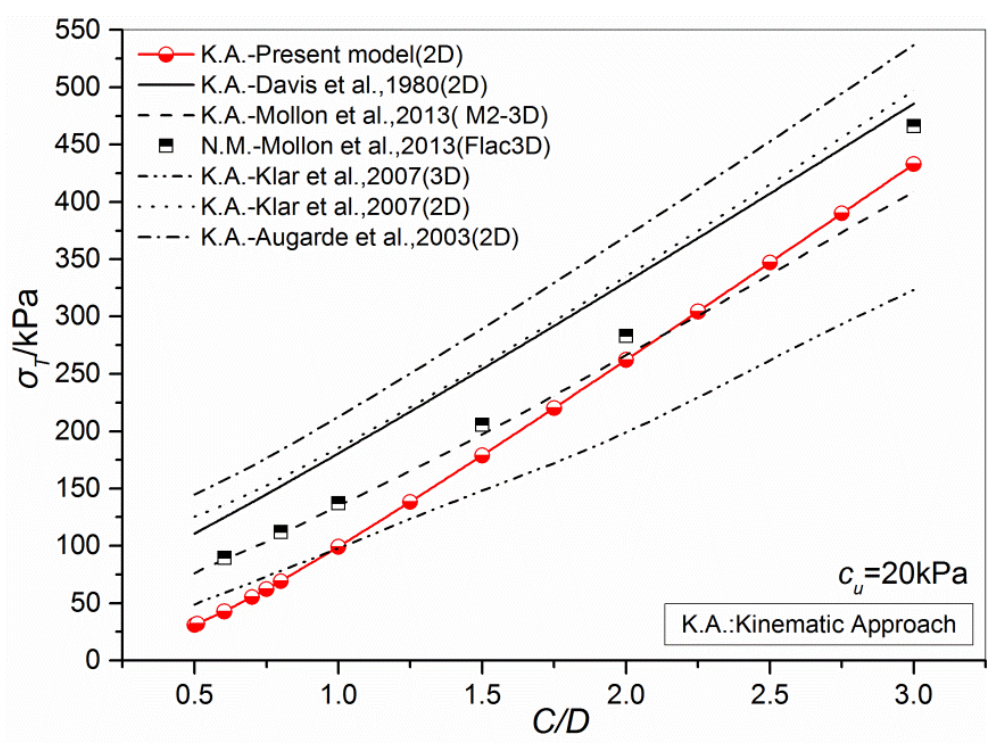


(a) $c_{u}=20 \mathrm{kPa}$

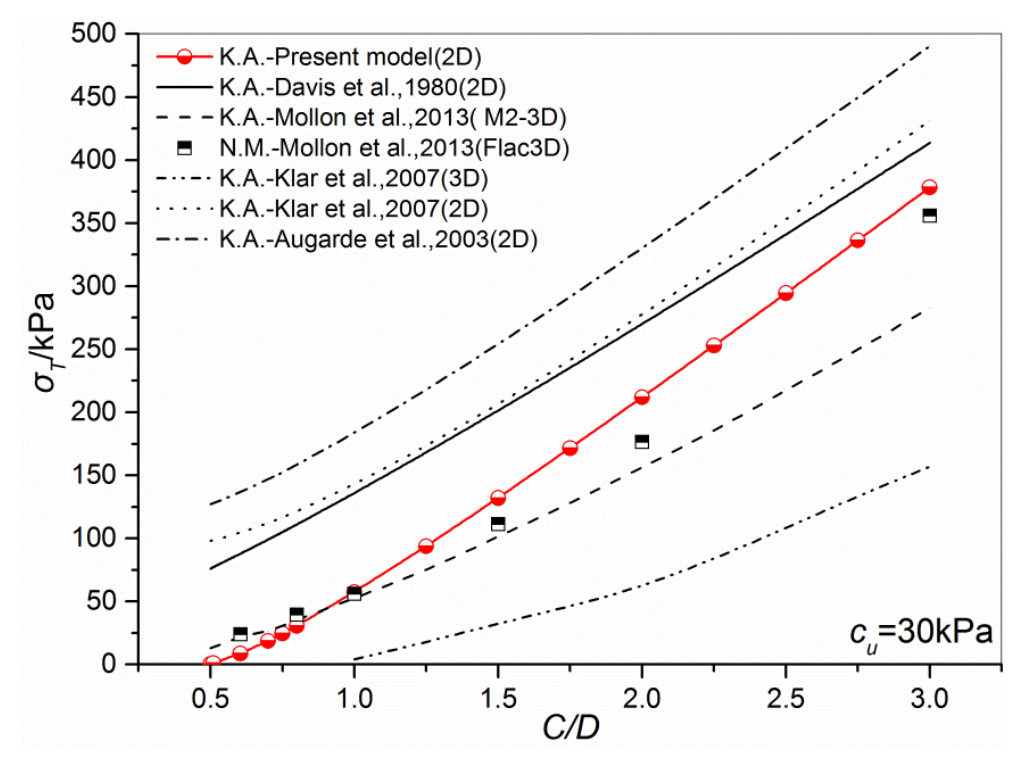

(b) $c_{u}=30 \mathrm{kPa}$

\section{Fig.17 Comparisons of collapse pressures of various of models}

Figure 17 shows that the solutions proposed by the present model are better than these proposed by other 2D models. In Figure 17(a), when $\gamma D / c_{u}$ is 9.0, the values of $\sigma_{\mathrm{T}}$ provided by the present model compares the better with the numerical model for $C / D>1.5$, whereas the values of $\sigma_{\mathrm{T}}$ provided by present model compares the worse with the numerical model for $\mathrm{C} / \mathrm{D} \leq 1.5$. It can be seen from Figure 17(b) that the values of $\sigma_{\mathrm{T}}$ provided by the present model are very satisfying for the values of $\mathrm{C} / \mathrm{D}$ from 0.5 to 3.0 for $\gamma D / c_{u}=6.0$. The reason is that the increase of the soil undrained cohesion weakens an effect to the critical collapse pressure of the tunnel face due to three-dimensional equilibrium conditions. Since the yield criterion, normality conditions (e.g. associate flow rule), and the vertical soil arching effect are considered in the present model, each failure zone in front of the tunnel face has clear physical concept and the present model can explain well the failure mechanism of the tunnel face driven by shield.

Figure 18 compares the failure zones of the present mechanism and that proposed by Davis et al. (1980) for various values of $C / D$. 


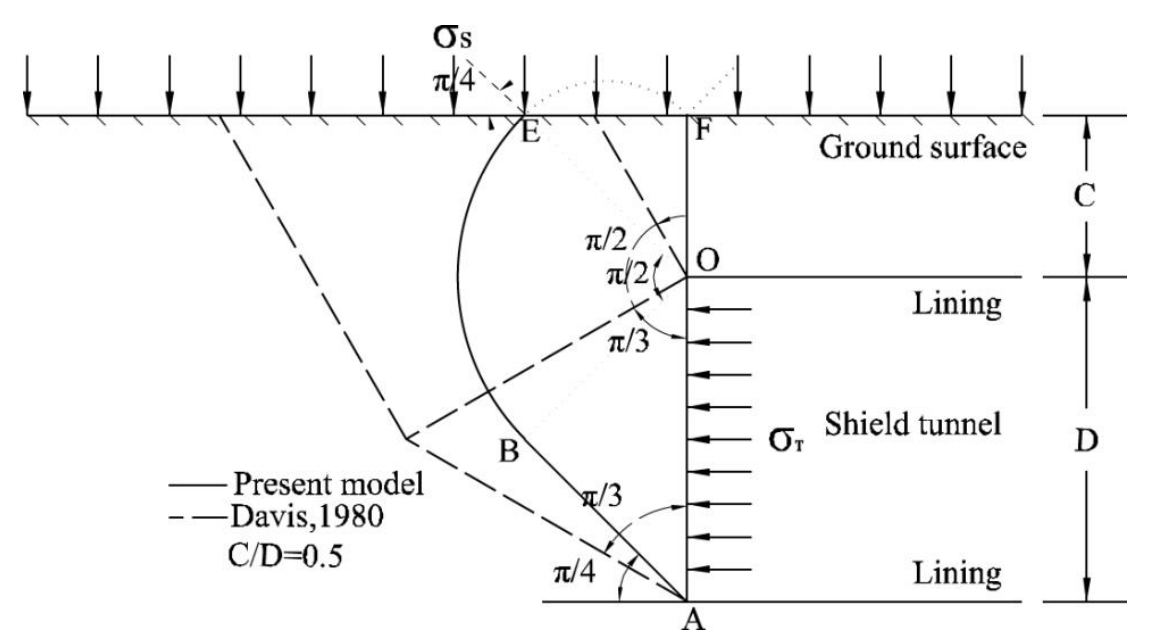

(a) $\mathrm{C} / \mathrm{D}=0.5$

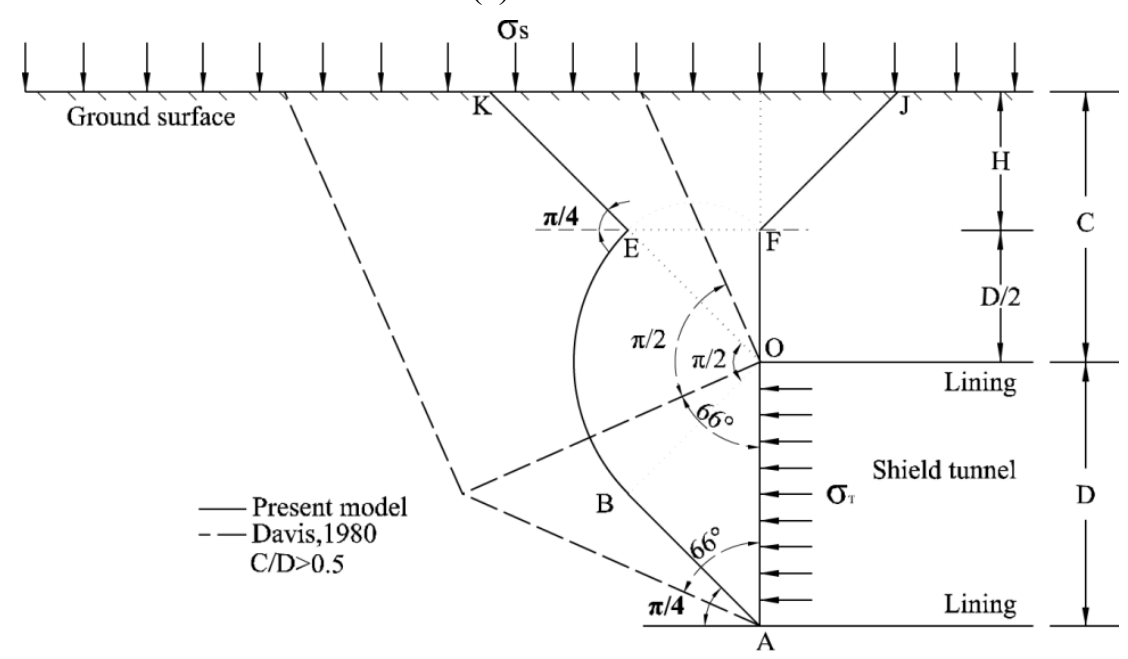

(b) $\mathrm{C} / \mathrm{D}=1.0$

Fig. 18 Comparisons of failure zones between the present mechanism and that proposed by Davis under different $C / D$ values: (a) $C / D=0.5$; (b) $C / D=1.0$

Figure 18 shows that the size of the fracture zone obtained using the present model is much smaller than that obtained by Davis et al. (1980). This is because this study considers the influence of the vertical soil arching effect and the normality conditions, whereas the fracture mechanism proposed by Davis et al. (1980) neglects the normality condition. The fracture mechanism of the present model is consistent with the experimental result obtained by Schofield (1980), as shown in Figure 18(b). Based on the limit analysis and the slip-line theories and together with considering the normality condition and yield criterion, the reasonable size of the fracture zone in front of the tunnel face is obtained in this study. The proposed failure mechanism significantly improves the existing upper-bound solutions for the face stability of circular tunnels in purely cohesive soils.

\section{Conclusions}

Based on the kinematic approach of the limit analysis and slip-line theories, a new 2D analytical 26 
model to assess the critical collapse pressure of a circular tunnel is proposed. This model considers the vertical soil arching effect and the normal condition. The upper-bound solutions proposed by the present model are compared with the existing classical solutions of two-dimension or threedimension models including analytical and numerical solutions. The main conclusions are presented as follows.

(1) The shape of a new 2D analytical model proposed by this study depends on the values of $C / D$. When the value of $C / D$ is 0.5 , the new analytical model consists of three zones, i.e., zones I, II and III. When the value of $C / D$ is greater than 0.5 , it may consist of four zones, i.e., zones I, II, III and possibly IV. Whether or not zone IV is considered depends on the values of the dimensionless parameters $\gamma D / c_{u}, \sigma_{S} / c_{u}$ and $C / D$.

(2) The comparisons between the present model and the existing classical solutions of $2 \mathrm{D}$ or $3 \mathrm{D}$ models show that the present model significantly improves the best existing upper-bound solutions of the collapse. As the present failure mechanism satisfies the normality conditions and considers the vertical soil arching effect at the top of zone III, the size of the fracture zone in front of the tunnel face given by the present model is far less than that given by the existing models. Thus, the tunnel pressure required to maintain stability obtained by the present model may be lower than those obtained by the existing models with increasing values of soil weight parameter $\gamma \mathrm{D} / c_{u}$.

(3) The contribution of the cohesion is greater than that of the soil weight and the surcharge loading for the critical collapse pressure of the tunnel face when $C / D$ is lower than 3.0; furthermore, the effect gradually weakens with increasing $C / D$. The contribution of the surcharge loading on the ground surface for the collapse loads is independent of the values of $C / D$. The critical coefficient $N_{\gamma}$ (i.e., the effect of the soil weight) increases linearly with increasing $C / D$. The difference in the critical coefficient $N_{\gamma}$ between the present model and the classical load factor is 0.4862 . This shows that the contribution of the soil weight in the tunnel for the critical collapse pressure of the tunnel face can't be ignored in the present model: it is $48.6 \%$ of the soil weight contribution when $C / D$ is equal to 0.5 . 
(4) In this study, the present model is a 2D analysis model in which certain assumptions are made. Therefore, to establish a more accurate 2D analysis model, or even a 3D analysis model, further investigations are required for purely cohesive soils.

\section{Acknowledgements}

The authors acknowledge the financial support provided by the National Natural Science Foundation of China (Grant Nos.41572275),the Natural Science Foundation of Shandong Province(Grant No. ZR2018MEE051 ), and the Project of Shandong Province Higher Educational Science and Technology Program (Grant No. J15LG01).

\section{References}

Augarde,C.E., Lyamin, A.V., and Sloan, S.W.(2003). Stability of an undrained plane strain heading revisited. Computers and Geotechnics,30(5),419-430.

Berthoz,N., Branque, D., Subrin, D., Wong, H., and Humbert, E.(2012). Face failure in homogeneous and stratified soft ground:Theoretical and experimental approaches on 1g EPBS reduced scale model. Tunnelling and Underground Space Technology.,30(4),25-37.

Broms,B.B., and Bennermark, H.(1967). Stability of clay at vertical openings. J.Soil Mech. Found. Eng.,193, SM1, 71-94.

Chen, R.P., Tang, L.J., Ling, D.S.,and Chen, Y.M.(2011). Face stability analysis of shallow shield tunnels in dry sandy ground using the discrete element method. Comput. Geotech., 38(2), 187-195.

Chen, R.P., Li, J., Kong, L.G., and Tang, L.J.(2013). Experimental study on face instability of shield tunnel in sand. Tunnelling and Underground Space Technology.,33(1),12-21.

Chen, W.F.(1975). Limit analysis and soil plasticity. Elsevier: Amsterdam.

Chevalier, B., Takano, D., and Otani, J.(2013). Comparison of X-ray CT and discrete element method in the evaluation of tunnel face failure. GeoX2010:Advances in computed Tomography for Geomaterials, John Wiley \& Sons, Inc.,397-405.

Davis, E.H., Gunn, M.J., Mair, R.J., and Seneviratne, H.N.(1980). The stability of shallow tunnels and underground openings in cohesive material. Géotechnique.,30(30),397-416. 
Ellstein, A.R.(1986). Heading failure of lined tunnels in soft soils. Tunnels and Tunnelling., $18(6), 51-54$

Ewing, D.J.F., Hill, R.(1967). The plastic constraint of V-notched tension bars. J.Mech. Phys. Solids, $15,115-124$.

Gunn, M.J.(1980). A note on the centred-fan stress field and its use in plasticity problems relevant to geotechnical engineering. Cambridge University Engineering Department Internal Report.

Idinger, G., Aklik, P., Wu, W., Borja, RI.(2011) Centrifuge model test on the face stability of shallow tunnel. Acta Geotechnica, 6(2), 105-117.

Kimura, T., and Mair, R.J.(1981). Centrifugal testing of model tunnels in clay. Proc., 10th Int. Conf. on Soil Mechanics and Foundation Engineering, Stockholm, Rotterdam: Balkema, Vol.1, 319-322.

Kirsch,A.(2010). Experimental investigation of the face stability of shallow tunnels in sand. Acta Geotech.5(1),43-62.

Klar, A., Osman, A.S., and Bolton, M.(2007). 2D and 3D upper bound solutions for tunnel excavation using 'elastic' flow fields. International Journal for Numerical and Analytical Methods in Geomechanics., 31,(12),1367-1374.

Lu,X.L., Wang, H.R., Huang, M.S.(2014). Upper bound solution for the face stability of shield tunnel below the water table. Mathematical Problems in Engineering . 1:1-11.

Leca, E., and Dormieux, L.(1990). Upper and lower bound solutions for the face stability of shallow circular tunnels in frictional material. Géotechnique., 40(4),581-606.

Lee, C.J., Wu, B.R., Chen, H.T., and Chiang, K.H.(2006). Tunneling stability and arching effects during tunneling in soft clayey soil. Tunnelling and Underground Space Technology., 21(2), $119-132$.

Mollon, G., Dias,D., and Soubra, A.H.(2009). Probabilistic analysis and design of circular tunnels against face stability. International Journal of Geomechanics.,ASCE. 9(6), 237-249.

Mollon, G., Dias, D., and Soubra, A.H.(2010). Face stability analysis of circular tunnels driven by a pressurized shield. Journal of Geotechnical and Geoenvironmental Engineering., ASCE. $136(1), 215-229$ 
Mollon, G., Dias, D., and Soubra, A.H.(2011). Rotational failure mechanisms for the face stability analysis of tunnels driven by pressurized shields. International Journal for Numerical and Analytical Methods in Geomechanics., 35(12),1363-1388.

Mollon, G., Dias, D., and Soubra, A.H.(2013). Continuous velocity fields for collapse and blowout of a pressurized tunnel face in purely cohesive soil. International Journal for Numerical and Analytical Methods in Geomechanics., 37,(13),2061-2083.

Schofield, A.N.(1980). Cambridge geotechnical centrifuge operations. Géotechnique.,30, (3), 227268.

Senent,s.,Mollon,G.,Jimenez.,R.(2013).Stability of tunnel face in rock masses with the Hoek-Brown failure criterion. International Journal of Rock Mechanics \& Mining Sciences. 60,440-451.

Senent. S., Jimenez, R.(2015). A tunnel face failure mechanism for layered ground, considering the possibility of partial collapse. Tunnelling and Underground Space Technology. 47, 182-192.

Sloan S.W., Assadi A. Undrained stability of a plane strain heading. Canadian Geotechnical Journal 1994;31:443-500.

Zhang, C.P., Han, K.H., and Zhang, D.L.(2015). Face stability analysis of shallow circular tunnels in cohesive-frictional soils. Tunnelling and Underground Space Technology., 50,345-357.

Zhang, Z. X., Hu, X.Y., Scott, K.D.(2011). A discrete numerical approach for modeling face stability in slurry shield tunneling in soft soils. Computers \& Geotechnics. 38(1), 94-104. 